\title{
Ritonavir acts synergistically with panobinostat to enhance histone acetylation and inhibit renal cancer growth
}

\author{
AKINORI SATO, TAKAKO ASANO, MAKOTO ISONO, KEIICHI ITO and TOMOHIKO ASANO \\ Department of Urology, National Defense Medical College, Tokorozawa, Saitama 359-8513, Japan
}

Received April 16, 2014; Accepted June 12, 2014

DOI: $10.3892 / \mathrm{mco} .2014 .349$

\begin{abstract}
There is currently no curative treatment for advanced renal cancer. Enhancing histone acetylation is a promising epigenetic-based therapy for cancer; however, in solid tumors, the efficacy of histone deacetylase (HDAC) inhibitors alone is limited. The human immunodeficiency virus protease inhibitor ritonavir is also a CYP3A4 inhibitor and we hypothesized that combining ritonavir with the HDAC inhibitor panobinostat, one of the substrates of CYP3A4, may effectively eliminate renal cancer cells by enhancing the activity of panobinostat. The combination of ritonavir and panobinostat synergistically inhibited cancer cell growth and cancer cell colony formation, while only slightly inhibiting the growth of renal proximal tubule epithelial cells. This combination significantly induced apoptosis in cancer cells and this apoptosis was considered to be caspase-dependent, since the pan-caspase inhibitor Z-VAD-FMK reduced the number of Annexin V-positive cells. In murine subcutaneous xenograft models using Caki-1 cells, a 10-day treatment with the combination of ritonavir and panobinostat significantly inhibited tumor growth. Panobinostat alone increased histone acetylation in a dose-dependent manner and the co-administration of ritonavir synergistically enhanced this acetylation. Furthermore, this combination inhibited the expression of HDACs, which may also play a role in the enhancement of histone acetylation. Thus, the present study may provide a basis for testing the combination of ritonavir and panobinostat for patients with advanced renal cancer.
\end{abstract}

\section{Introduction}

A novel approach to the treatment of advanced renal cancer is needed, as, despite the wide use of kinase inhibitors or mammalian target of rapamycin inhibitors, there is currently no curative treatment. Increasing histone acetylation is an attractive epigenetic approach to cancer treatment and panobi-

Correspondence to: Dr Akinori Sato, Department of Urology, National Defense Medical College, 3-2 Namiki, Tokorozawa, Saitama 359-8513, Japan

E-mail: zenpaku@ndmc.ac.jp

Key words: ritonavir, panobinostat, histone acetylation, renal cancer nostat is a novel histone deacetylase (HDAC) inhibitor that has been shown to exert beneficial antitumor effects in phase II trials in patients with hematological malignancies $(1,2)$. Panobinostat was also clinically tested in patients with solid tumors $(3,4)$, but no complete or partial response was observed in those studies. Ritonavir is a human immunodeficiency virus protease inhibitor widely used for the treatment of acquired immune deficiency syndrome, which is also a potent CYP3A4 inhibitor (5). As panobinostat is one of the substrates of CYP3A4 (6), we hypothesized that ritonavir may enhance the activity of panobinostat by increasing its intracellular accumulation through inhibiting its degradation.

In the present study, we aimed to evaluate the combined effect of ritonavir and panobinostat on renal cancer cells in vitro and in vivo and investigate their mechanism of action.

\section{Materials and methods}

Cell lines. The Caki-1, ACHN, 769-P and 786-O renal cancer cell lines were purchased from the American Type Culture Collection (Rockville, MD, USA) and renal proximal tubule epithelial cells (RPTECs) were purchased from Lonza (Basel, Switzerland). The cells were cultured in either minimum essential medium, Dulbecco's modified Eagle's medium, RPMI-1640 or renal epithelial cell basal medium (depending on the cell line) supplemented with $10 \%$ fetal bovine serum and $0.3 \%$ penicillin/streptomycin (Invitrogen, Carlsbad, CA, USA) at $37^{\circ} \mathrm{C}$ under $5 \% \mathrm{CO}_{2}$ in a humidified incubator.

Reagents. Panobinostat, purchased from Cayman Chemical Company (Ann Arbor, MI, USA) and ritonavir, purchased from Toronto Research Chemicals (Toronto, ON, Canada), were dissolved in dimethyl sulfoxide and stored at $-20^{\circ} \mathrm{C}$ until use. The pan-caspase inhibitor Z-VAD-FMK was purchased from Enzo Life Sciences (Farmingdale, NY, USA).

Cell viability assay. Starting one day after $5 \times 10^{3}$ cells were seeded into a 96-well culture plate, they were cultured for $48 \mathrm{~h}$ in medium containing 10,25 or $50 \mathrm{nM}$ panobinostat and/or 25 or $50 \mu \mathrm{M}$ ritonavir. Cell viability was then evaluated with the MTS assay (CellTiter 96 AQueous kit; Promega, Madison, WI, USA) according to the manufacturer's protocol.

Colony formation assay. For the colony formation assay, 100 individual cells were seeded in 6-well plates 1 day prior to 
treatment and treated with $25 \mathrm{nM}$ panobinostat and/or $50 \mu \mathrm{M}$ ritonavir for $48 \mathrm{~h}$. The cells were then given fresh media and cultured for 1-2 weeks. The colonies were fixed with $100 \%$ methanol, stained with Giemsa's solution and counted.

Murine xenograft model. The efficacy of the combination of ritonavir and panobinostat in vivo was assessed using the murine subcutaneous xenograft model. The procedures were performed according to a protocol approved by the Institutional Animal Care and Use Committee. Ten million Caki-1 cells were implanted subcutaneously in nude mice purchased from CLEA Japan, Inc. (Tokyo, Japan) and treatment was initiated 7 days later (day 1), when all the mice exhibited measurable tumors. The mice were divided into control and treatment groups ( $n=5$ per group). The treated mice received intraperitoneal injection of either panobinostat ( $2 \mathrm{mg} / \mathrm{kg})$, or ritonavir $(50 \mathrm{mg} / \mathrm{kg})$, or both, while the control mice received vehicle only. The injections were given once a day, 5 days a week, for 2 weeks. Tumor growth was measured using a digital caliper and tumor volume was calculated as volume $=0.5 \times$ length $\mathrm{x}$ width ${ }^{2}$.

Annexin $V$ assay. Induction of apoptosis was assayed using the Annexin V assay. Briefly, $1.5 \times 10^{5}$ cells were seeded in a 6 -well culture plate 1 day prior to treatment. The cells were then cultured in medium containing $25 \mathrm{nM}$ panobinostat and/or $50 \mu \mathrm{M}$ ritonavir for $48 \mathrm{~h}$, stained with Annexin V according to the manufacturer's protocol (Beckman Coulter, Marseille, France) and analyzed by flow cytometry using the CellQuest Pro software (BD Biosciences, San Jose, CA, USA). To evaluate whether the apoptosis induced by the combination of ritonavir and panobinostat was caspase-dependent, the cells were treated with $25 \mathrm{nM}$ panobinostat combined with $50 \mu \mathrm{M}$
Table I. Combination indices for ritonavir and panobinostat in renal cancer cells.

\begin{tabular}{lccc}
\hline & \multicolumn{3}{c}{ Panobinostat $(\mathrm{nM})$} \\
\cline { 2 - 4 } Ritonavir $(\mu \mathrm{M})$ & 10 & 25 & 50 \\
\hline Caki-1 & & & \\
25 & 0.963 & 0.735 & 1.074 \\
50 & 0.652 & 0.155 & 0.309 \\
ACHN & & & \\
25 & 0.858 & 0.874 & 1.108 \\
50 & 1.034 & 0.852 & 0.177 \\
$769-\mathrm{P}$ & & & \\
25 & 0.874 & 1.001 & 0.550 \\
50 & 0.758 & 0.458 & 0.224 \\
$786-\mathrm{O}$ & & & \\
25 & 1.456 & 1.245 & 0.921 \\
50 & 1.204 & 0.775 & 0.081 \\
\hline
\end{tabular}

ritonavir, with or without $40 \mu \mathrm{M}$ Z-VAD-FMK. After $48 \mathrm{~h}$, induction of apoptosis was evaluated with the Annexin V assay using flow cytometry.

Western blotting. The changes in protein expression induced by the combination were evaluated using western blotting. The cells were treated under the indicated conditions for $48 \mathrm{~h}$ and whole-cell lysates were obtained using radioimmunoprecipitation assay buffer. The proteins were resolved on $12.5 \%$ SDS-polyacrylamide gels and electrophoretically transferred

$\mathbf{A}$
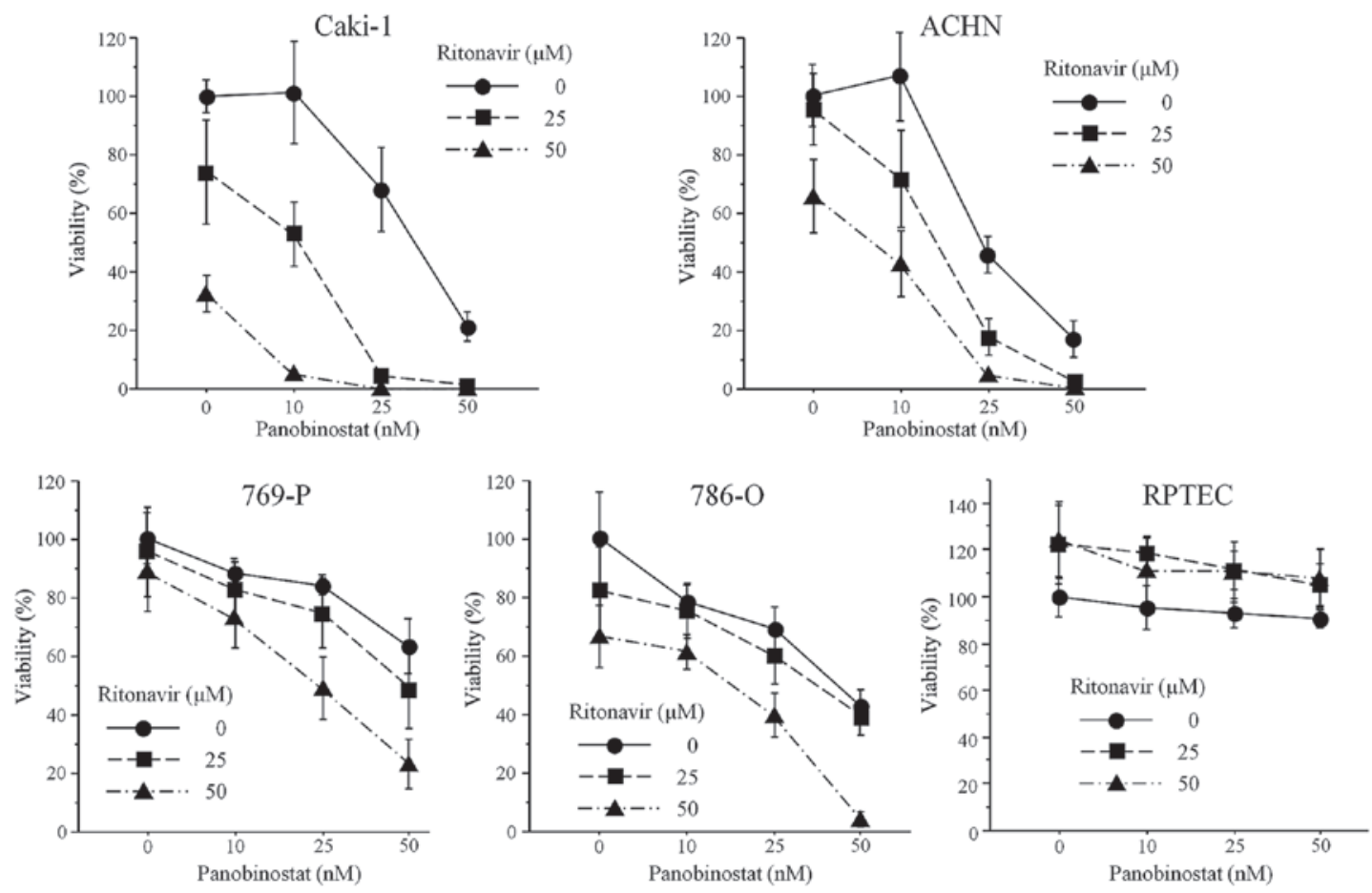

Figure 1. The combination of ritonavir and panobinostat synergistically inhibited renal cancer cell growth, while only slightly inhibiting renal proximal tubule epithelial cells (RPTECs). (A) MTS assay after a 48-h treatment ( $n=6$, mean \pm SD). 
B

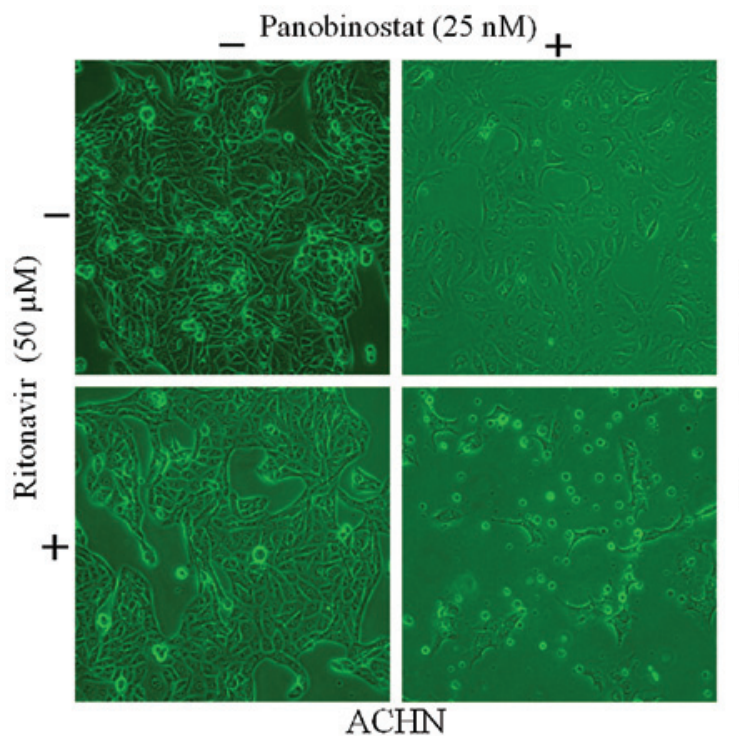

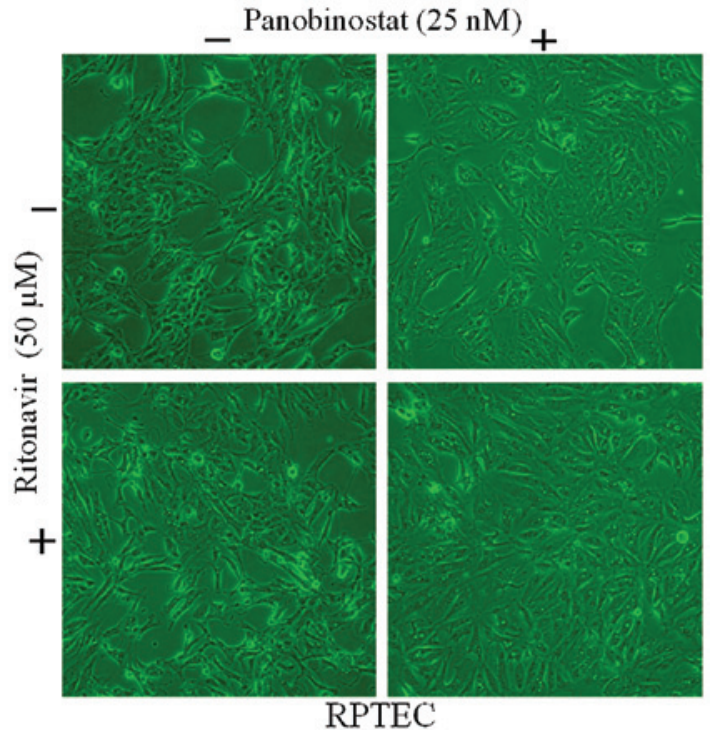

RPTEC
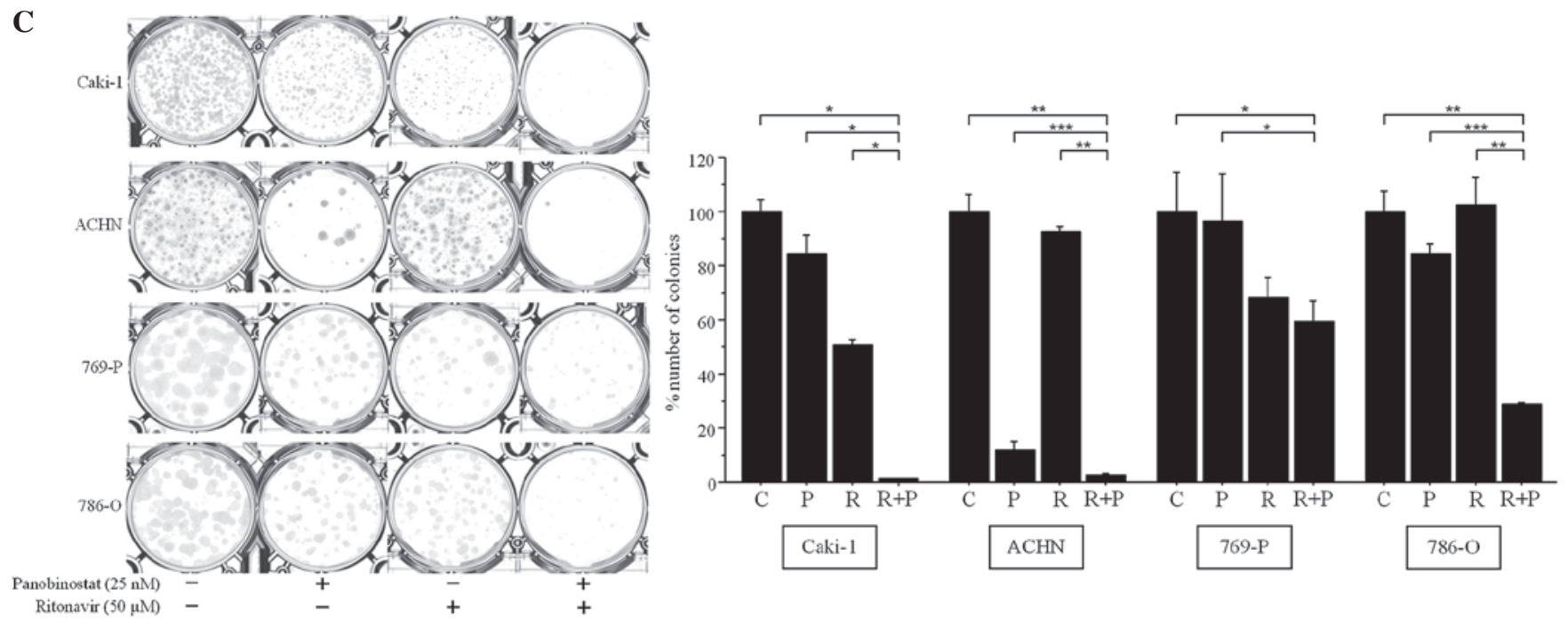

D

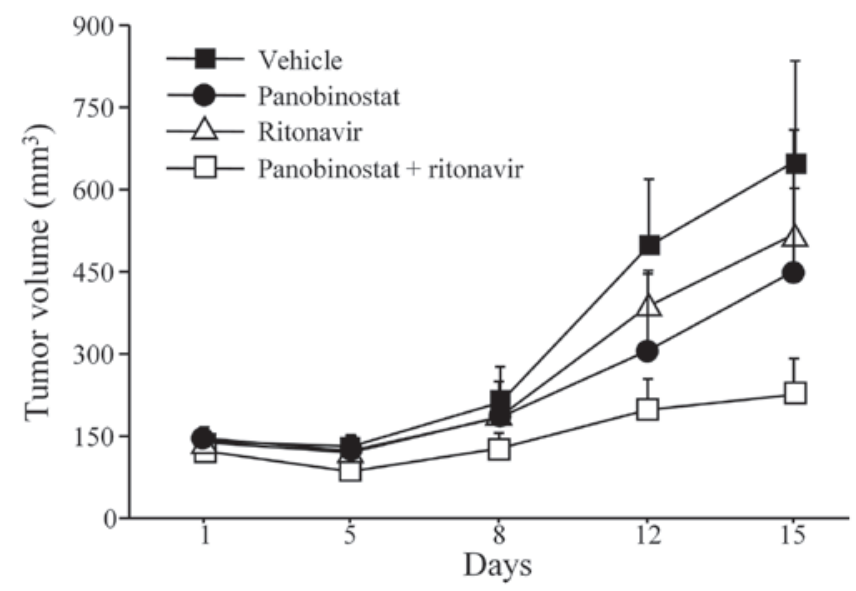

Figure 1. Continued. The combination of ritonavir and panobinostat synergistically inhibited renal cancer cell growth, while only slightly inhibiting renal proximal tubule epithelial cell (RPTEC) growth. (B) Photomicrographs of ACHN cells and RPTECs after a 48-h treatment (original magnification, x100). The majority of the ACHN cells treated with the combination are floating. (C) Colony formation assay. The cells were treated for $48 \mathrm{~h}$ under the indicated conditions and allowed to grow for $1-2$ weeks. C, control; $\mathrm{P}, 25 \mathrm{nM}$ panobinostat; and $\mathrm{R}, 50 \mu \mathrm{M}$ ritonavir. ${ }^{*} \mathrm{P}=0.0495,{ }^{* *} \mathrm{P}=0.0463,{ }^{* * *} \mathrm{P}=0.0431$. (D) The combination significantly suppressed tumor growth in vivo. A murine xenograft model was established using Caki-1 cells. The control group received intraperitoneal injections of dimethyl sulfoxide and the treatment groups received $2 \mathrm{mg} / \mathrm{kg}$ panobinostat, or $50 \mathrm{mg} / \mathrm{kg}$ ritonavir, or both. The injections were given once a day, 5 days a week for 2 weeks. $\mathrm{P}=0.0472$ at day 12 .

to nitrocellulose membranes. After the membranes were blocked by $5 \%(\mathrm{w} / \mathrm{v})$ skimmed milk according to standard procedures, they were first incubated with either anti-acetylated histone (Abcam, Cambridge, UK), anti-HDAC1, 
A
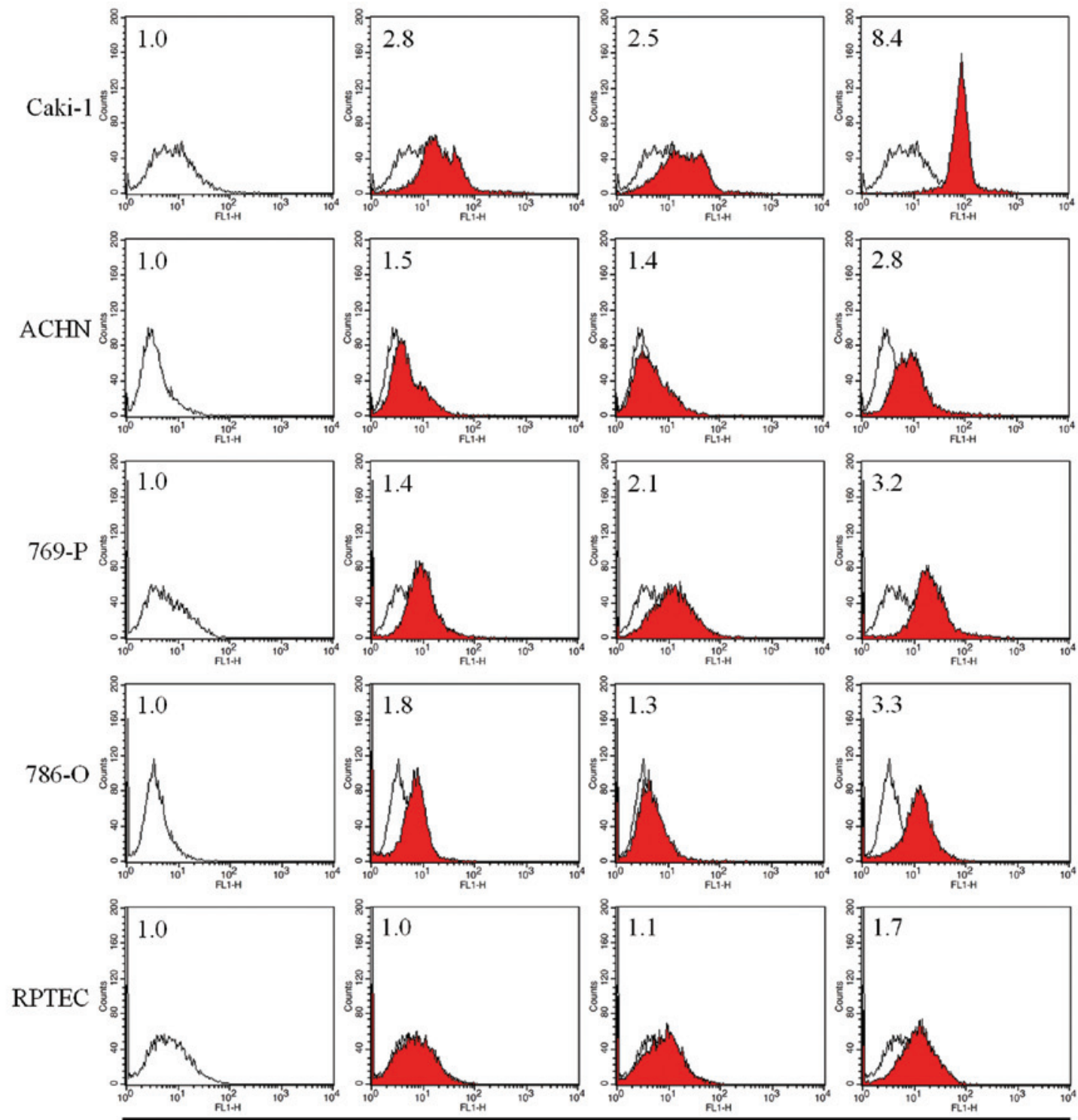

Panobinostat $(25 \mathrm{nM})$ -

Ritonavir $(50 \mu \mathrm{M})$

B

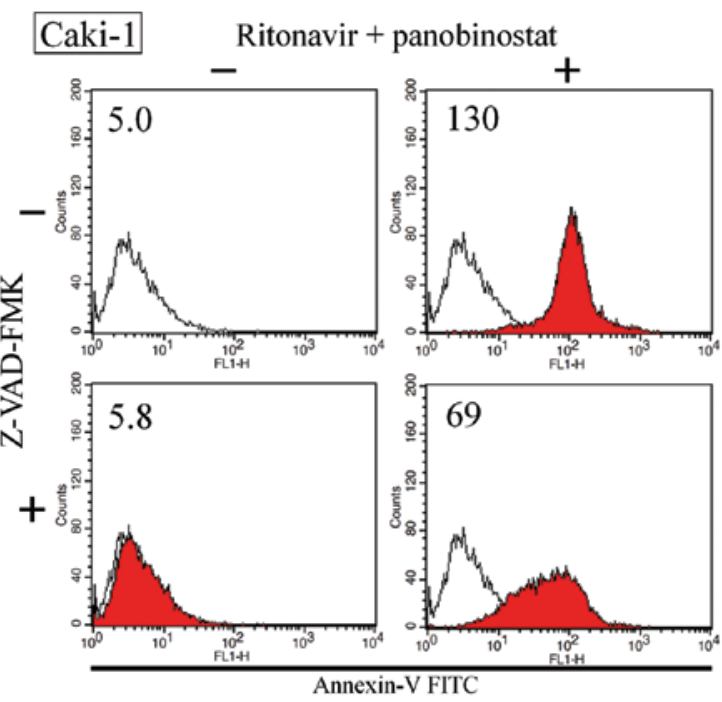

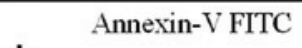

$+$

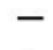

$+$

$+$

$+$

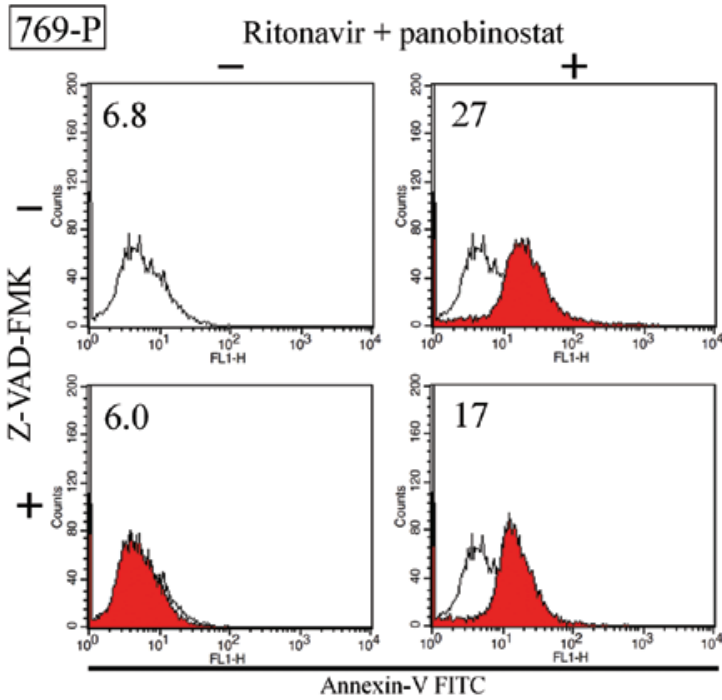

Figure 2. The combination of ritonavir and panobinostat induced apoptosis. (A) Annexin V assay. The cells were treated under the indicated conditions for $48 \mathrm{~h}$. White, control; red, treated cells. The inset in each graph is the relative Annexin V-fluorescein isothiocyanate (FITC) fluorescence intensity (control=1). (B) Annexin V assay after a 48 -h treatment with the combination of $50 \mu \mathrm{M}$ ritonavir, $25 \mathrm{nM}$ panobinostat and $40 \mu \mathrm{M}$ Z-VAD-FMK, a pan-caspase inhibitor. White, control; red, treated cells. The inset in each graph is the Annexin V-FITC fluorescence intensity. Representative results are shown. RPTEC, renal proximal tubule epithelial cells. 
A

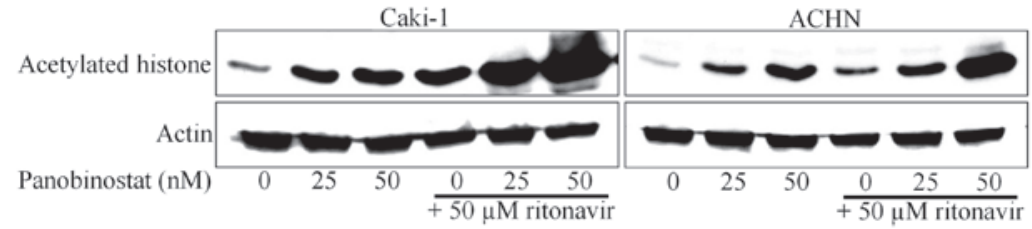

769-P 786-O

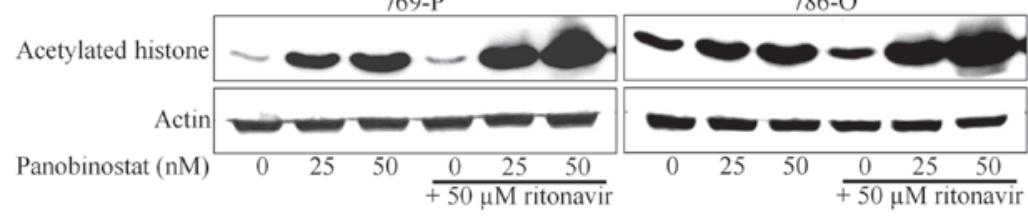

RPTEC

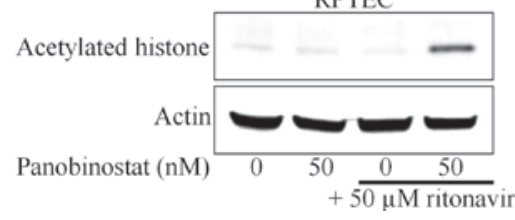

Caki-1

ACHN

B

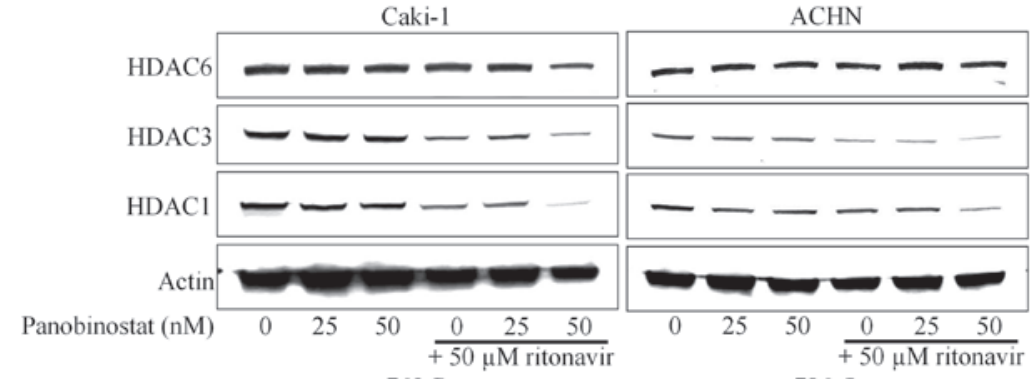

769-P 786-O

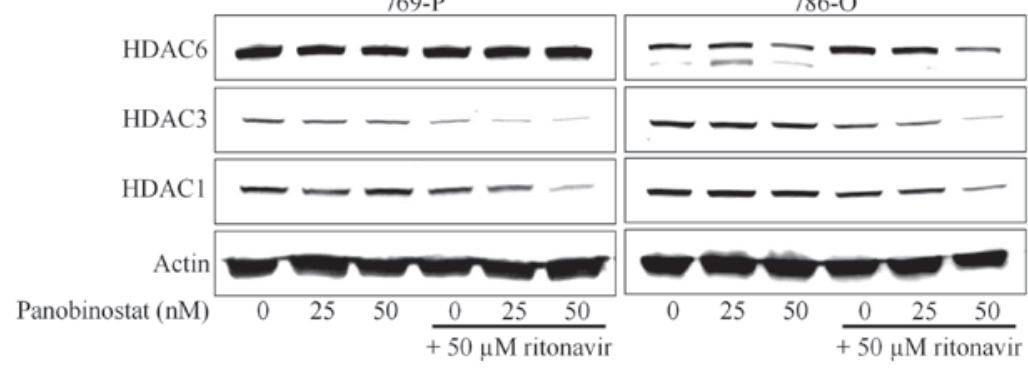

Figure 3. Representative western blots (with actin as a loading control) demonstrating that a 48-h treatment with ritonavir and panobinostat synergistically (A) induced histone acetylation and (B) decreased the expression of histone deacetylases (HDACs). RPTEC, renal proximal tubule epithelial cells.

anti-HDAC3, anti-HDAC6 (Santa Cruz Biotechnology, Santa Cruz, CA, USA) or anti-actin (Millipore, Billerica, MA, USA) primary antibodies and subsequently with horseradish-tagged secondary antibodies (Bio-Rad, Hercules, CA, USA). The bands were visualized by chemiluminescence with the ECL Plus system (GE Healthcare, Wauwatosa, WI, USA) according to the manufacturer's instructions.

Statistical analysis. CalcuSyn software (Biosoft, Cambridge, UK) was used for calculating the combination indices. The statistical significance of observed differences between samples was determined using the Mann-Whitney U test (StatView software; SAS Institute, Cary, NC, USA). P<0.05 was considered to indicate a statistically significant difference.

\section{Results}

Ritonavir and panobinostat synergistically inhibited renal cancer cell growth. According to the MTS assay, the combination of panobinostat and ritonavir effectively inhibited the growth of renal cancer cells, while only slightly inhibiting the growth of RPTECs (Fig. 1A). The morphological changes in the renal cancer cells were notable (the majority of the cells treated by the combination were floating), while there was no apparent change in the RPTECs (Fig. 1B). Using the Chou-Talalay method, an isobologram analysis was performed and combination indices were calculated, demonstrating that the combined effect on cell growth was synergistic (combination indices $<1$ ) under most treatment conditions (Table I). We then investigated whether the combination of ritonavir and panobinostat affects the clonogenicity of renal cancer cells. The colony formation assay revealed that this combination significantly inhibited the clonogenic survival of renal cancer cells (Fig. 1C). Thus, the combination of ritonavir with panobinostat was found to be effective in inhibiting renal cancer cell growth in vitro.

Ritonavir combined with panobinostat significantly suppressed renal cancer cell growth in a murine xenograft model. Following the in vitro experiments, we evaluated the efficacy of the combination of ritonavir and panobinostat 
in vivo. In murine xenograft tumor models, a 10-day treatment with ritonavir and panobinostat was well tolerated and significantly suppressed tumor growth $(\mathrm{P}=0.0472$ at day 12$)$ (Fig. 1D). The average tumor size at day 15 was $652 \pm 184 \mathrm{~mm}^{3}$ (mean \pm standard error) in the vehicle-treated mice and $220 \pm 67 \mathrm{~mm}^{3}$ in the combination-treated mice.

Ritonavir combined with panobinostat induced apoptosis. The combination of ritonavir and panobinostat significantly increased Annexin V-fluorescein isothiocyanate (FITC) fluorescence intensity in renal cancer cells and was thus shown to induce apoptosis. In accordance with the results of the MTS assay, this combination induced apoptosis only slightly in RPTECs (Fig. 2A). We then investigated whether the combination-induced apoptosis was caspase-dependent. In Caki-1 and 769-P cells, co-incubation with the pan-caspase inhibitor Z-VAD-FMK markedly reduced the degree to which the combination increased Annexin V-FITC fluorescence intensity (Fig. 2B), indicating that the combination-induced apoptosis was indeed caspase-dependent.

Ritonavir enhanced histone acetylation induced by panobinostat. We next evaluated whether ritonavir enhanced the activity of panobinostat. Since panobinostat is an HDAC inhibitor, we hypothesized that the degree of induction of histone acetylation reflects its activity. Panobinostat alone increased histone acetylation in a dose-dependent manner and ritonavir synergistically enhanced this acetylation (Fig. 3A). Thus, ritonavir was shown to enhance the activity of panobinostat in renal cancer cells. Of note, in RPTECs, even $50 \mathrm{nM}$ panobinostat failed to induce histone acetylation and the combined effect of ritonavir and panobinostat was weaker compared to that in renal cancer cells. This is consistent with the results of the MTS and the Annexin V assays. Interestingly, this combination decreased the expression of HDACs (Fig. 3B), which may also play a role in enhancing histone acetylation.

\section{Discussion}

Targeted therapy using kinase inhibitors and mammalian target of rapamycin inhibitors is a mainstay in the treatment of metastatic renal cancer; however, a new treatment approach is needed, as, although these inhibitors increase progression-free survival to some extent, they are not curative.

The acetylation and deacetylation of histones is crucial in the modulation of chromatin structure (7). The levels of histone acetylation are determined by the balance between the activities of histone acetyltransferases and HDACs (8) and deacetylation of histones tightens their interaction with DNA, leading to a closed chromatin structure that inhibits gene transcription (9). HDACs are associated with a number of cellular oncogenes and tumor suppressor genes (10); thus, compounds targeting HDACs have attracted significant attention as anticancer drugs (11). Panobinostat is one such compound that has been clinically tested in patients with refractory metastatic renal cell carcinoma (4). In that study, however, panobinostat was not found to be effective. Since panobinostat is deactivated by CYP3A4 (6), we hypothesized that inhibiting this drug-eliminating machinery may enhance the activity of panobinostat.
As expected, the combination of ritonavir and panobinostat significantly induced apoptosis and synergistically inhibited renal cancer cell growth, as shown by combination indices of $<1$ under most treatment conditions. Of note, this combination induced minimal apoptosis in RPTECs and only slightly inhibited their growth, suggesting that it is advantageous in terms of side effects, despite its drastic anticancer cell effects.

Panobinostat caused histone acetylation and this acetylation was enhanced by ritonavir, which is consistent with the hypothesis that ritonavir enhances the activity of panobinostat. Furthermore, in RPTECs, even treatment with $50 \mathrm{nM}$ panobinostat failed to cause histone acetylation and the acetylation-enhancing effect of the combination appeared to be weaker compared to that in cancer cells. This result suggests that normal epithelial cells tolerate panobinostat well and the present combination therapy acts more specifically against renal cancer cells. Interestingly, we also observed that the combination decreased the expression of HDACs, which may be another important mechanism underlying its enhancement of histone acetylation. This decreased HDAC expression may also be a consequence of the enhanced histone acetylation, as HDAC inhibitors themselves may decrease the expression of HDACs $(12,13)$.

Although the enhancement of panobinostat activity is an important mechanism of action of ritonavir, the combination of the two is considered to inhibit cancer growth by diverse mechanisms. In the present study, ritonavir itself exhibited antiproliferative activity against renal cancer cells, suggesting that it may not only act as a CYP3A4 inhibitor. Ritonavir was recently shown to exert antitumor effects through the inhibition of proteins such as nuclear factor- $\kappa \mathrm{B}(14)$ and heat shock protein (HSP) 90 (15) and it was also reported to inhibit renal cancer growth by inhibiting heat shock factor 1, a transcription factor of HSP 90, when used in combination with 17-allylamino-17-demethoxygeldanamycin (16). Furthermore, the inhibition of HDACs, particularly HDAC6, acetylates HSP 90 and suppresses its function as a molecular chaperone (17). It is considered that the combination of ritonavir and panobinostat may cooperatively suppress HSP 90, causing unfolded protein accumulation and, thereby, endoplasmic reticulum stress. However, further study is required to prove this mechanism.

The combination of ritonavir and panobinostat may be one of the candidates for a clinical trial in patients with advanced renal cancer. However, as CYP3A4 is also a major liver enzyme catalyzing drug metabolism, there is a major concern that ritonavir may increase the serum concentration of panobinostat excessively and cause severe adverse events. However, a clinical study using panobinostat with ketoconazole as a CYP3A4 inhibitor, demonstrated that the combination increased the maximum concentration (Cmax) of panobinostat 1.6-fold and the area under the curve 1.8-fold, without significantly altering the time required to reach Cmax or the half-life (6). The authors of that study concluded that co-administration of panobinostat with CYP3A inhibitors is feasible, as the increases in the parameters of panobinostat pharmacokinetics were not clinically relevant. In addition, considering our results that the combination of ritonavir and panobinostat was not associated with lethal side effects in vivo and affected the growth of RPTECs only slightly, the side effects of this combination are expected to be minimal. Optimal concentrations, 
however, must be carefully determined in phase I trials with strict monitoring of the drugs' serum concentrations.

In conclusion, ritonavir enhanced the activity of panobinostat and the combination of the two synergistically inhibited renal cancer growth. The inhibition of the expression of HDACs by this combination may further enhance histone acetylation. To the best of our knowledge, this is the first study demonstrating the beneficial combined effect of ritonavir and panobinostat on renal cancer cells and it may provide a basis for clinical studies with this combination in patients with advanced renal cancer.

\section{References}

1. Younes A, Sureda A, Ben-Yehuda D, et al: Panobinostat in patients with relapsed/refractory Hodgkin's lymphoma after autologous stem-cell transplantation: results of a phase II study. J Clin Oncol 30: 2197-2203, 2012.

2. Ghobrial IM, Campigotto F, Murphy TJ, et al: Results of a phase 2 trial of the single-agent histone deacetylase inhibitor panobinostat in patients with relapsed/refractory Waldenström macroglobulinemia. Blood 121: 1296-1303, 2013.

3. Morita S, Oizumi S, Minami H, et al: Phase I dose-escalating study of panobinostat (LBH589) administered intravenously to Japanese patients with advanced solid tumors. Invest New Drugs 30: 1950-1957, 2012.

4. Hainsworth JD, Infante JR, Spigel DR, et al: A phase II trial of panobinostat, a histone deacetylase inhibitor, in the treatment of patients with refractory metastatic renal cell carcinoma. Cancer Invest 29: 451-455, 2011.

5. Eagling VA, Back DJ and Barry MG: Differential inhibition of cytochrome $\mathrm{P} 450$ isoforms by the protease inhibitors, ritonavir saquinavir and indinavir. Br J Clin Pharmacol: 190-194, 1997.

6. Hamberg P, Woo MM, Chen LC,Verweij J, Porro MG, Zhao L, Li W, van der Biessen D, Sharma S, Hengelage T and de Jonge M: Effect of ketoconazole-mediated CYP3A4 inhibition on clinical pharmacokinetics of panobinostat (LBH589), an orally active histone deacetylase inhibitor. Cancer Chemother Pharmacol 68 805-813, 2011.
7. Marks P, Rifkind RA, Richon VM, Breslow R, Miller T and Kelly WK: Histone deacetylases and cancer: causes and therapies. Nat Rev Cancer 1: 194-202, 2001.

8. Wade PA: Transcriptional control at regulatory checkpoints by histone deacetylases: molecular connections between cancer and chromatin. Hum Mol Genet 10: 693-698, 2001.

9. Grunstein M: Histone acetylation in chromatin structure and transcription. Nature 389: 349-352, 1997.

10. Cress WD and Seto E: Histone deacetylases, transcriptional control, and cancer. J Cell Physiol 184: 1-16, 2000.

11. Yoo CB and Jones PA: Epigenetic therapy of cancer: past, present and future. Nat Rev Drug Discov 5: 37-50, 2006.

12. Hrzenjak A, Moinfar F, Kremser ML et al: Valproate inhibition of histone deacetylase 2 affects differentiation and decreases proliferation of endometrial stromal sarcoma cells. Mol Cancer Ther 5: 2203-2210, 2006.

13. Gui CY, Ngo L, Xu WS, Richon VM and Marks PA: Histone deacetylase (HDAC) inhibitor activation of p21WAF1 involves changes in promoter-associated proteins, including HDAC1. Proc Natl Acad Sci USA 101: 1241-1246, 2004.

14. Dewan MZ, Tomita M, Katano H, et al: An HIV protease inhibitor, ritonavir targets the nuclear factor-kappaB and inhibits the tumor growth and infiltration of EBV-positive lymphoblastoid B cells. Int J Cancer 124: 622-629, 2009.

15. Srirangam A, Mitra R, Wang M, et al: Effects of HIV protease inhibitor ritonavir on Akt-regulated cell proliferation in breast cancer. Clin Cancer Res 12: 1883-1896, 2006.

16. Sato A, Asano T, Ito K and Asano T: 17-allylamino-17-demethoxygeldanamycin and ritonavir inhibit renal cancer growth by inhibiting the expression of heat shock factor-1. Int J Oncol 41: 46-52, 2012.

17. Bali P, Pranpat M,Bradner J, et al: Inhibition of histone deacetylase 6 acetylates and disrupts the chaperone function of heat shock protein 90: a novel basis for antileukemia activity of histone deacetylase inhibitors. J Biol Chem 280: 26729-26734, 2005. 